APS

physics

This is the accepted manuscript made available via CHORUS, the article has been published as:

\title{
Observation of Coherent Helimagnons and Gilbert Damping in an Itinerant Magnet
}

J. D. Koralek, D. Meier, J. P. Hinton, A. Bauer, S. A. Parameswaran, A. Vishwanath, R.

Ramesh, R. W. Schoenlein, C. Pfleiderer, and J. Orenstein

Phys. Rev. Lett. 109, 247204 - Published 12 December 2012

DOI: 10.1103/PhysRevLett.109.247204 


\title{
Observation of Coherent Helimagnons and Gilbert Damping in an Itinerant Magnet
}

\author{
J. D. Koralek, ${ }^{1, *}$ D. Meier, ${ }^{2, *}$ J. P. Hinton,,${ }^{1,2}$ A. Bauer, ${ }^{3}$ S. A. Parameswaran, ${ }^{2}$ A. \\ Vishwanath, ${ }^{1,2}$ R. Ramesh, ${ }^{1,2}$ R. W. Schoenlein, ${ }^{1}$ C. Pfleiderer, ${ }^{3}$ and J. Orenstein ${ }^{1,2}$ \\ ${ }^{1}$ Materials Science Division, Lawrence Berkeley National Laboratory, Berkeley, California 94720, USA \\ ${ }^{2}$ Department of Physics, University of California, Berkeley, California 94720, USA \\ ${ }^{3}$ Physik Department E21, Technische Universität München, D-85748 Garching, Germany
}

(Dated: Dated October 24, 2012)

\begin{abstract}
We study the magnetic excitations of itinerant helimagnets by applying time-resolved optical spectroscopy to $\mathrm{Fe}_{0.8} \mathrm{Co}_{0.2} \mathrm{Si}$. Optically excited oscillations of the magnetization in the helical state are found to disperse to lower frequency as the applied magnetic field is increased; the fingerprint of collective modes unique to helimagnets, known as helimagnons. The use of time-resolved spectroscopy allows us to address the fundamental magnetic relaxation processes by directly measuring the Gilbert damping, revealing the versatility of spin dynamics in chiral magnets.
\end{abstract}

The concept of chirality pervades all of science, having profound implications in physics, chemistry and biology alike. In solids, relativistic spin-orbit coupling can give rise to the Dzyaloshinskii-Moriya (DM) interaction [1,2], imparting a tendency for the electron spins to form helical textures with a well-defined handedness in crystals lacking inversion symmetry. Helical spin order is especially interesting when the magnetism arises from the same electrons responsible for conduction as is the case in doped FeSi which displays unconventional magnetoresitence [3, 4], helimagnetism [6], and the recently discovered Skyrmion lattice [7, 9]. The excitations of helimagnets have been studied over the past 30 years, culminating recently in a comprehensive theory of spin excitations called helimagnons [10, 11]. Signatures of helimagnons have been observed in neutron scattering [12] and microwave absorption [13], yet little is known about their magnetodynamics and relaxation phenomena on the sub-picosecond timescales on which magnetic interactions occur. Understanding the dynamics, however, is of great importance regarding spin transfer torque effects in chiral magnets, and related proposed spintronics applications [14-16].

In this work we study the dynamics of collective spin excitations in the itinerant helimagnet $\mathrm{Fe}_{0.8} \mathrm{Co}_{0.2} \mathrm{Si}$. Our optical pump-probe measurements identify anomalous modes at zero wavevector $(q=0)$ which we identify unmistakably as helimagnons. These helimagnons manifest as strongly damped magnetization oscillations that follow a characteristic scaling relation with respect to temperature and magnetic field. The sub-picosecond time resolution of our technique enables determination of the intrinsic Gilbert damping parameter which is found to be an order of magnitude larger than in localized systems, revealing the versatility of the spin-lattice interactions available in the emergent class of DM-driven helimagnets.

Despite being a non-magnetic insulator, $\mathrm{FeSi}$ is transformed into an itinerant magnet upon doping with cobalt [3, 17]. We have chosen $\mathrm{Fe}_{0.8} \mathrm{Co}_{0.2} \mathrm{Si}$ for our study because it can easily be prepared in high quality single crystals $[18,19]$ with a reasonably high magnetic ordering temperature $T_{c} \approx$ $30 \mathrm{~K}$, and its exotic equilibrium properties are well character- ized, opening the door for non-equilibrium dynamical studies. Small-angle neutron scattering $[8,9]$ was used to determine the phase diagram and has revealed helimagnetic spin textures below $T_{c}$ that emerge from the interplay between the ferromagnetic exchange and DM interactions. In zero magnetic field the spins form a proper helix with a spatial period of $\approx 350 \AA$ [20], whereas finite fields cant the spins along the helix wavevector, $\mathbf{k}_{h}$, (see Fig. 1(c)) inducing a conical state with a net magnetization. Sufficiently high fields, $H \geq H_{c}$, suppress the conical order in favor of field alignment of all spins. In the experiments reported here, femtosecond pulses of linearly polarized $1.5 \mathrm{eV}$ photons from a Ti:Sapphire oscillator were used to excite a (110) oriented single crystal of $\mathrm{Fe}_{0.8} \mathrm{Co}_{0.2} \mathrm{Si}$ at near normal incidence. The changes induced in the sample by the pump pulse were probed by monitoring the reflection and Kerr rotation of time-delayed probe pulses from the same laser. In order to minimize laser heating of the sample the laser repetition rate was reduced to $20 \mathrm{MHz}$ with an electro-optic pulse picker. The pump-pulse energy was $10 \mu \mathrm{J} / \mathrm{cm}^{2}$ and the polarization was directed along the crystallographic (001) axis of the sample, however, no dependence on the pump pulse polarization was observed. Signal to noise was improved by modulation of the pump beam at $100 \mathrm{KHz}$ and synchronous lock-in detection of the reflected probe. Kerr rotation was measured using a Wollaston prism and balanced photodiode. All temperature and field scans presented in this work were performed from low to high $T$ and $\mathbf{H} \|(110)$ after zero-field cooling.

Fig. 1 shows the transient reflectivity, $\Delta R / R$, as a function of temperature and magnetic field. At high temperature we observe a typical bolometric response from transient heating of the sample by the pump pulse (Fig. 1(a)) [21]. This is characterized by a rapid increase in reflectivity, followed by twocomponent decay on the fs and ps timescales, corresponding to the electron-phonon thermalization time and subsequent thermal diffusion into the bulk. We discuss laser heating effects in detail in the supplementary material and conclude that after $100 \mathrm{ps}$ the sample temperature recovers to less than $8 \mathrm{~K}$ above the base temperature and that no average heating occurs. As the sample is cooled below $50 \mathrm{~K}$, the small thermal 

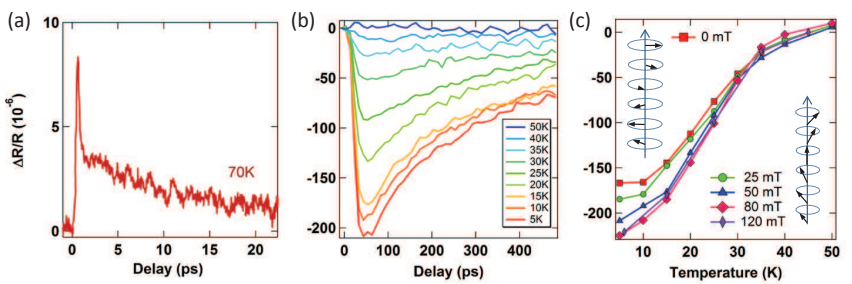

FIG. 1. Time dependence of the pump-induced transient reflectivity $\Delta R / R$ in the (a) paramagnetic and (b) helimagnetic states. The temperature dependence of the maximum $\Delta R / R$ is plotted in (c) for several applied magnetic fields. Also shown in (c) are illustrations of the helical (left) and conical (right) spin structures present in the low temperature magnetic phases for $H<H_{c}$ (see phase diagram in Fig. 2(e)).

signal is beset by a much larger negative reflectivity transient (Fig. 1(b)) with a decay time of roughly $\tau_{R} \approx 175$ ps at low temperature (Fig. 3(b) inset). The resulting temperature dependence of the peak $\Delta R / R$ values is plotted in Fig. 1(c) for several applied fields. We attribute the observed behavior to the strong coupling between the spin and charge degrees of freedom in $\mathrm{Fe}_{0.8} \mathrm{Co}_{0.2} \mathrm{Si}$. Here the magnetic order suppresses the conductivity of the sample, which is seen in resistivity measurements as a sign change in $\partial \rho / \partial T$ discussed in detail in Reference [23]. The magnetically driven upturn in $\rho$ coincides with the onset of $\Delta R / R$, both exhibiting the same low temperature behavior as shown in the supplemental material. We therefore conclude that the weakly field dependent $\Delta R / R$ is dominated by the pump pulse weakening the magnetic order, suppressing the conductivity, and shifting spectral weight to low energy.

To access the magnetization dynamics more directly we analyze the polarization state of the probe pulses, which rotates by an angle $\theta_{\mathrm{K}}$ upon reflection from the sample surface, in proportion to the component of the magnetization along the light trajectory. The change in Kerr rotation induced by the optical pump, $\Delta \theta_{\mathrm{K}}$, is shown in Fig. 2 as a function of temperature and field. The upper panels show temperature scans at fixed magnetic field, while a field scan at fixed temperature is shown in panel (d). We observe that $\Delta \theta_{\mathrm{K}}$ changes sign as $H$ is reversed (not shown), and vanishes as $H$ goes to zero or as temperature is raised. Two distinct magnetodynamic regimes are clearly distinguishable in the data; purely exponential behavior for $T_{c} \lesssim T \lesssim 50 \mathrm{~K}$ with oscillations emerging for $T<T_{c}$.

In order to analyze the magnetization dynamics, we use a simple phenomenological function that separates the oscillatory and non-oscillatory components seen in the data. The full details of the fitting procedure are given in the supplemental material. The key component of our fitting function is a decaying sinusoidal oscillation,

$$
\Delta \theta_{\mathrm{K}}=e^{-\frac{t}{\tau_{\mathrm{K}}}}[A+B \sin (\omega t)]
$$
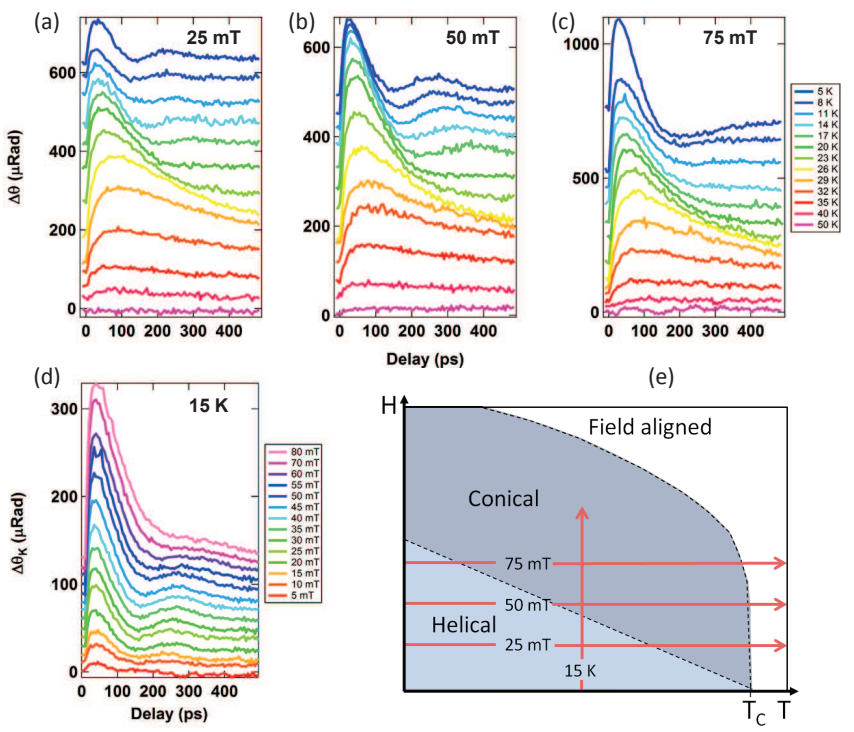

Delay (ps)

(e)

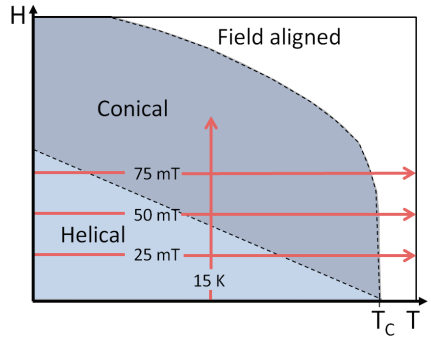

FIG. 2. (a),(b),(c) Time dependence of the pump-induced change in Kerr rotation, $\Delta \theta_{\mathrm{K}}$, as a function of temperature for several applied magnetic fields. (d) $\Delta \theta_{\mathrm{K}}$ as a function of magnetic field at $T=15 \mathrm{~K}$. Curves are offset for clarity. Also shown is a schematic phase diagram (e), adapted from Reference [9], with red arrows illustrating the temperature and field scans used in (a)-(d).

with a time dependent frequency,

$$
\omega(t)=2 \pi f_{0}\left[1+D\left(e^{-\frac{t}{\tau_{\mathrm{K}}}}\right)\right]
$$

which decays to a final value $\omega_{0}=2 \pi f_{0}$. Note that $D$ is a phenomenological parameter which characterizes the strength of the time-dependence and which remains fixed across all $T$ and $H$ in our analysis. We emphasize that there is only a single decay time $\tau_{\mathrm{K}}$ describing the magneto dynamics, and it is directly related to the Gilbert damping parameter $\alpha=\left(2 \pi f_{0} \tau_{\mathrm{K}}\right)^{-1}$. Our fitting function produces excellent fits to the data as illustrated in Fig. 3(a), allowing accurate extraction of the oscillation frequencies and decay times shown in Figs. 3(b-d). The oscillation frequency is reduced as either field or temperature is increased, while the decay time $\tau_{\mathrm{K}}$ is roughly constant and equal to $\tau_{R}$ below $T_{c}$. As the temperature is raised towards the phase transition, the relaxation time $\tau_{\mathrm{K}}$ diverges, which is consistent with a diverging magnetic correlation length due to the presence of a critical point. The similarity between the decay times $\tau_{R}$ and $\tau_{\mathrm{K}}$ within the ordered phase reflects strongly correlated charge and spin degrees of freedom, and supports the notion that $\Delta R / R$ is determined by the magnetic order. However, from the data of the inset of Fig. 3(b) it is not clear whether this strong spin-charge correlation persists as we approach the phase transition.

The magnetic oscillation frequency reaches $f_{0} \approx 4.8 \mathrm{GHz}$ at low temperature, which corresponds to a Larmor precession of spins subjected to a field of roughly $170 \mathrm{mT}$, which is comparable to the critical field $H_{c}$ required to destroy the spin helix. This, together with the fact that the oscillation fre- 

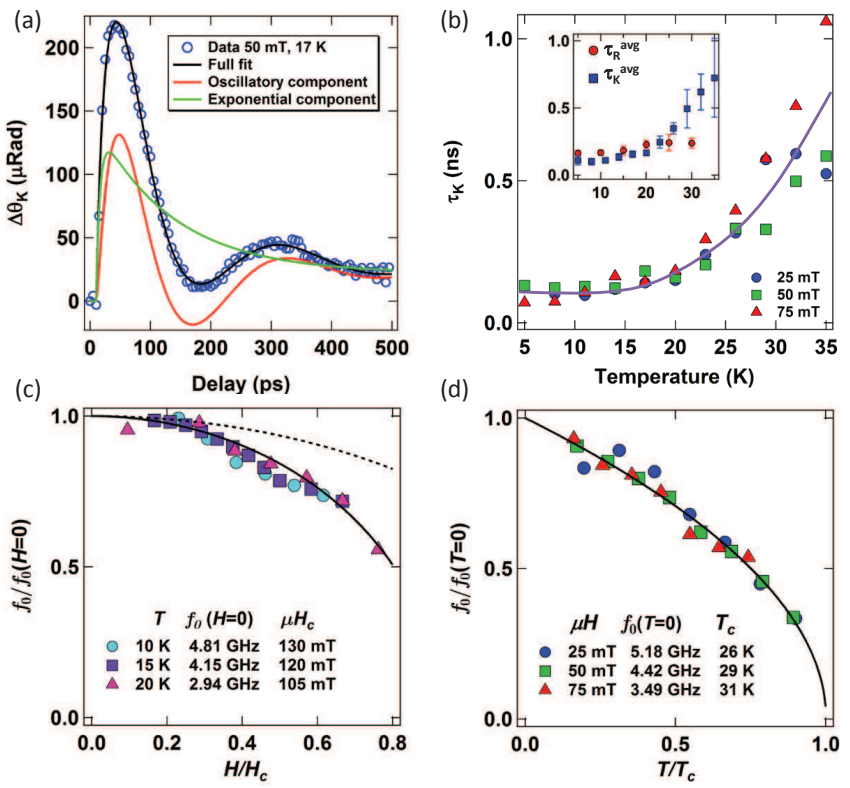

(d)

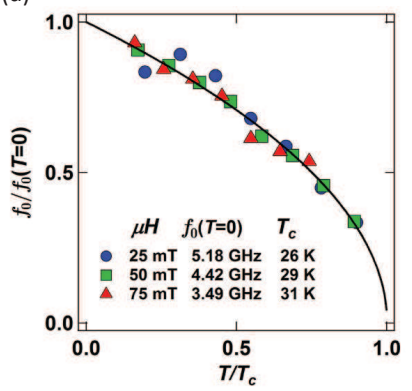

FIG. 3. (a) Exemplary $\Delta \theta_{\mathrm{K}}$ oscillation data (blue circles) and fit (black line) using the model described in the text and fitting procedure detailed in the supplemental information. The fit is decomposed into an exponential term (green curve) and an oscillatory term (red curve). The fitting function uses a single time constant $\tau_{\mathrm{K}}$ for all terms which is plotted in panel (b) as a function of temperature for different magnetic fields. The solid line is a guide to the eye. The inset to (b) compares $\tau_{\mathrm{K}}$ to the reflectivity decay time, $\tau_{R}$, both averaged over all fields, with error bars showing the standard deviation. Panels (c) and (d) show the reduced magnetization oscillation frequency for field scans and temperature scans respectively. The values of $H_{c}$ are extracted from AC susceptibility measurements [31], and the values and $T_{c}$ are deduced from the Kerr measurements as described in the text. The dotted and solid lines in (c) and (d) are derived from the theory as described in the main text. Statistical error from the fitting is smaller than the data markers in (b-d).

quency is nonzero only in the helical state, suggests that the oscillations are coming from excitations unique to the helical structure. It is well known that magnetization oscillations can be optically induced by ultrafast generation of coherent magnons [26-28], however, ordinary magnons cannot explain our data as their frequency would increase with $H$, opposite to what is seen in Fig. 3(c).

Based on these observations, we propose the following interpretation of our results: In the helical magnetic phase, the pump photons weaken the magnetic order through the ultrafast demagnetization process [29]. This reduction in magnetic order gives rise to a decrease in the reflectivity at $1.5 \mathrm{eV}$ which is nearly field independent [23]. As a magnetic field is applied the spins become canted along the helix wavevector, giving rise to a macroscopic magnetization which we observe in Kerr rotation via its component along the probe light trajectory. The demagnetization from the pump is responsible for the initial peak seen in the $\Delta \theta_{\mathrm{K}}$ time traces, and is captured by the exponential component of our fitting function (green curve in Fig. 3(a)). The pump photons also launch a coherent spin wave, giving rise to the oscillations in $\Delta \theta_{\mathrm{K}}$ (red curve in Fig. 3(a)). The form of the oscillatory component goes like $\sin (\omega t)$ which, based on Reference [27], points towards impulsive stimulated Raman scattering as the mechanism of excitation. The anomalous field dependence shown in Fig. 3(c) leads to the unambiguous conclusion that the optically excited spin waves are the fundamental modes of helimagnets termed helimagnons [11]. Specifically, the optically accessible helimagnon mode consists of the constituents of the spin helix precessing in-phase about their local effective field. The ability to resolve helimagnons with femtosecond time resolution at $q=0$ is unique to our optical probe, and complements neutron scattering which is restricted to mapping helimagnon bands at higher $q$. This region of reciprocal space is particularly interesting in the case of helimagnets as the periodicity introduced by the helical spin texture generates bands that are centered at $q= \pm k_{h}$ and therefore have finite frequency modes at $q=0$ even in the absence of a gap. This is in contrast to ordinary magnons in which the bands are generally centered at $q=0$ so that the associated mode has zero frequency. We note that our observations are in agreement with previous work on the collective modes of skyrmions [30] which coexist with helimagnons in the so-called A-Phase as discussed in Reference [13]. The appearance of these modes is not expected in our data as their corresponding oscillation periods exceed the observed damping time in $\mathrm{Fe}_{0.8} \mathrm{Co}_{0.2} \mathrm{Si}$.

In order to quantitatively test the helimagnon interpretation we take the expression for the $q=0$ helimagnon frequency in an external magnetic field,

$$
f_{0}=g \mu_{\mathrm{B}} H_{c} \sqrt{1+\cos ^{2} \theta}
$$

where $g$ is the effective electron $g$-factor, $\mu_{\mathrm{B}}$ is the Bohr magneton, and $\frac{\pi}{2}-\theta$ is the conical angle, i.e. the amount the spins are canted away from $\mathbf{k}_{h}$. Ignoring demagnetization effects of the spin waves themselves, we can write $\sin \theta=\frac{H}{H_{c}}$, where $H_{c}$ is the critical field at which the spins all align with the field and the helimagnon ceases to exist as a well-defined mode. Within this simple model we obtain [10],

$$
f_{0}=g \mu_{\mathrm{B}} H_{c} \sqrt{1-\frac{1}{2}\left(\frac{H}{H_{c}}\right)^{2}}
$$

which expresses the magnon frequency as a function of applied field. This theory predicts the dotted line in Fig. 3(c), expressing the decrease in frequency with increasing $H$ which is unique to helimagnons. However, an even more pronounced field dependence is seen in the data, which can be captured by adjusting $H_{c}$ as $H_{c}^{*}=\gamma H_{c}$. The solid line in Fig. 3(c) is a fit yielding a value of $\gamma=0.66$ while keeping $H_{c}$ fixed to the values extracted from our AC susceptibility measurements (Fig. 3(c) legend). The need to introduce $H_{c}^{*}$ likely stems from the fact that Eq. (4) neglects effects such as demagnetization and anisotropies which necessarily alter the details of the field dependence. Nevertheless, the qualitative feature of a 
decrease in $f_{0}$ with $H$ and the good agreement with the functional form of the field dependence strongly favor the helimagnon interpretation.

The frequency $f_{0}$ also shows a pronounced temperature dependence which can be fit to the form $f_{0} \propto \sqrt{1-\left(T / T_{c}\right)}$, as illustrated by the solid line in Fig. 3(d). Here, $T_{c}$ is a fit parameter that is used to extrapolate the transition temperature since $f_{0}$ cannot be measured in the vicinity of the phase transition. Despite this limitation, the transition temperatures obtained from these fits are in reasonable agreement with neutron data [9]. An intuitive concept unifying the temperature and field dependence in Fig. 3(c-d) is that $f_{0}$ is determined by the spin components perpendicular to the helix wavevector which decrease with increasing $T$ and $H$.

The Gilbert damping parameter can be directly obtained from the measured decay times through the relation $\alpha=$ $\left(2 \pi f_{0} \tau_{\mathrm{K}}\right)^{-1}$, which gives an average value of $\alpha=0.4 \pm 0.1$ for the helimagnetic phase of $\mathrm{Fe}_{0.8} \mathrm{Co}_{0.2} \mathrm{Si}$. This is an order of magnitude larger than what was seen in insulating $\mathrm{Cu}_{2} \mathrm{OSeO}_{3}$ [13], where helimagnetism arises from localized rather than itinerant spins. The contrast in dynamics between these systems is critical in the context of potential spintronic applications based on helimagnetism where there is a tradeoff between fast switching which requires large damping, and stability which relies on low damping.

In summary, this work demonstrates ultrafast coherent optical excitation of spin waves in an itinerant DM-driven spin system and reveals the underlying spin dynamics. We identify these excitations as helimagnons through their anomalous field dependence and explain our observations with a comprehensive model. Our experiments directly yield the intrinsic Gilbert damping parameter, revealing a striking difference in spin relaxation phenomena between itinerant and localized helimagnets. The results elucidate the dynamics of collective modes common to the actively studied B20 transition metal compounds that codetermine their performance in potential spin based applications.

The work in Berkeley was supported by the Director, Office of Science, Office of Basic Energy Sciences, Materials Sciences and Engineering Division, of the U.S. Department of Energy under Contract No. DE-AC02-05CH11231. C.P. and A.B. acknowledge support through DFG TRR80 (From Electronic Correlations to Functionality), DFG FOR960 (Quantum Phase Transitions), and ERC AdG (291079, TOPFIT). A.B. acknowledges financial support through the TUM graduate school. D.M. acknowledges support from the Alexander von Humboldt foundation and S.A.P. acknowledges support from the Simons Foundation. C.P. and A.B. also thank S. Mayr, W. Münzer, and A. Neubauer. 
* These authors contributed equally to this work. email addresses: jdkoralek@1bl.gov and meier@berkeley.edu

[1] I. E. Dzyaloshinskii, Sov. Phys. JETP 5, 1259 (1957).

[2] T. Moriya, Phys. Rev. 120, 91 (1960).

[3] N. Manyala et al., Nature 404, 581 (2000).

[4] N. Manyala et al., Nature Mater. 3, 255 (2004).

[5] S. Blundell, Magnetism in Condensed Matter (Oxford Master Series in Physics) (2001).

[6] J. Beille, J. Voiron, and M. Roth, Solid State Commun. 47, 399 (1983).

[7] S. Mühlbauer et al., Science 323, 915 (2009).

[8] K. Ishimoto et al., Physica B 213 \& 214, 381 (1995).

[9] W. Münzer et al., Phys. Rev. B 81, 041203(R) (2010).

[10] M. Kataoka, J. Phys. Soc. Jap. 563635 (1987).

[11] D. Belitz, T. R. Kirkpatrick, and A. Rosch, Phys. Rev. B 3, 054431 (2006).

[12] M. Janoschek et al., Phys. Rev. B 81, 214436 (2010).

[13] Y. Onose et al., Phys. Rev. Lett. 109, 037603 (2012).

[14] K. Everschor et al., Phys. Rev. B 86, 054432 (2012).

[15] F. Jonietz et al., Science 330, 1648 (2010).

[16] T. Schulz et al., Nature Phys. 8, 301 (2012).

[17] G. Aeppli and Z. Fisk, Comments Condens. Matter Phys. 16, 155 (1992).

[18] W. Münzer, Diploma thesis, Technische Universität München, (2009).

[19] A. Neubauer et al., Rev. Sci. Instrum. 82, 013902 (2011).

[20] S. V. Grigoriev et al., Phys. Rev. Lett. 102, 037204 (2009).

[21] R. W. Schoenlein, W. Z. Lin, G. Fujimoto and G. L. Besley, Phys. Rev. Lett. 581680 (1987).

[22] S. I. Anisimov, B. L. Kapeliovich and T. L. Perelman, Sov. Phys. JETP 39, 375 (1975).

[23] F. P. Mena et al., Phys. Rev. B 73, 085205 (2006).

[24] S. A. Brazovskii, S. G. Dmitriev, Sov. Phys. JETP 42, 497 (1976).

[25] M. Janoschek et al., arXiv:1205.4780v1 (2012).

[26] M. van Kampen et al., Phys. Rev. Lett. 88, 227201 (2002).

[27] A. M. Kalashnikova et al., Phys. Rev. B 78, 104301 (2008).

[28] D. Talbayev et al., Phys. Rev. Lett. 101, 097603 (2008).

[29] A. Kirilyuk, A. V. Kimel, and T. Rasing, Rev. Mod. Phys. 82, 2731 (2010).

[30] M. Mochizuk, Phys. Rev. Lett. 108, 017601 (2012).

[31] A. Bauer et al. (unpublished) 